\title{
USES OF COLOURS: IN THEORY \& APPLICATION
}

\author{
Dr. Aekta Bisht
}

Assi. Prof.

Srinagar Garhwal

Drawing \& Painting, HNB Garhwal University

Pigments, colours and mediums are the tools of an artist. Ever since human beings stood upright, freeing their hands, they have been using natural pigments, earth colours and synthetic pigments to record their experiences. If the language is meant to communicate ideas and record events, then art was truly yhe first language.

Colour is the most immediate form of non- verbal communication. We naturally react to colour as we have evolved with a certain understanding of it, partly because the survival of our ancestors depended on it with regard to what to consume and avoid. Colour is used to represent thoughts and emotions in a way that no other element of design can, and it can act as an instant attention grabber whether in print, on screen or on a supermarket shelf. As such, colour is an important face og contemporary design. We all, inherently, have preferences that inform our decisions when dealing with colour, and we are subject to all the cultural norms and understandings of colour usage that surround us.

Colour is perhaps the first element that we register when we view something for the first time. Our cultural development and conditioning mean that we will naturally make associations based upon the colours we see, and these provide an idea of how we should react to an object or design that incorporates them. Colour attach meaning, and our reaction to this will depend upon cultural associations, trends, age and individual preferences.

\section{BASIC TERMINOLOGY}

Colour theory begins with the definition of different types of colour and how they can be combined for different uses. There are many colour theories available to the visual artist, among the most popular are Munsell, Ostwald and the Prang system. The Parang system is the one which is most likely taught in the schools.

Primary colours - Primary colours come in two forms: additive and subtractive. Additive primaries are red, blue and green that in the form of light combine to form white. The subtractive primary coloues or painting primaries are blue, red and yellow, which are associated with reflected light. When combined, the subtractive primary colours produce black. These colours are somewhat different from the four colour printing procedss where cyan, magenta and yellow together with black are used as the printing primaries because they produce a better result than painting primaries.

Secondary colours - A secondary set of colours can be produced by the combination to any two colours in equal proportion in the subtractive colour space, the secondary colours produced are red, green and blue, while in the additive colour space the secondary colours produced are cyan, magenta and yellow.

Tertiary colours - Tertiary colours are produced by combining a secondary colour with the primary colour that is not already present within it. This is equivalent to mixing subtractive primary 
colours in the proportions $2: 1$ or 1:2. Tertiary colours are not produced in this way in the fourcolour printing process as the addition of different amounts of colour accumulates and produces black. In colour theory primary colours are mixed to create secondary colours and tertiary colours can be made from these.
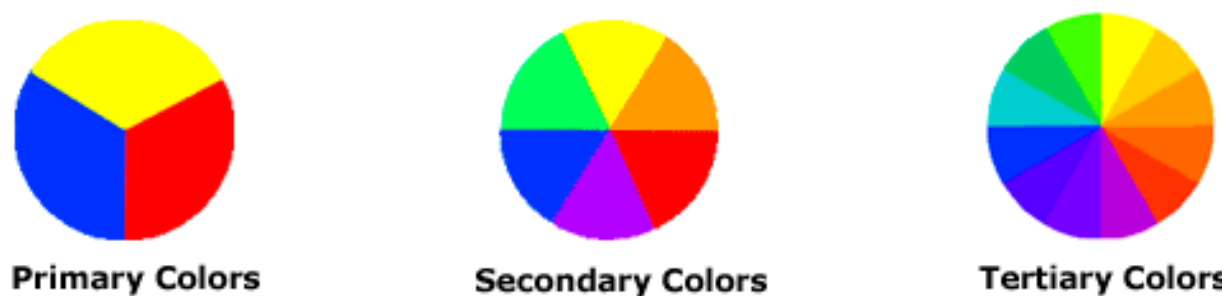

Color attributes were first understood in 19th century by physiologist Ewald Hering, His colour charts show how all colors arise from a combination of green-or-red, blue-or-yellow and brightness. (In his diagram, the green-neutral-red is vertical, and blue-neutral-yellow is horizontal.) The left circle shows relative mixtures of color attributes. The right circle shows what we perceive when these attributes are mixed.

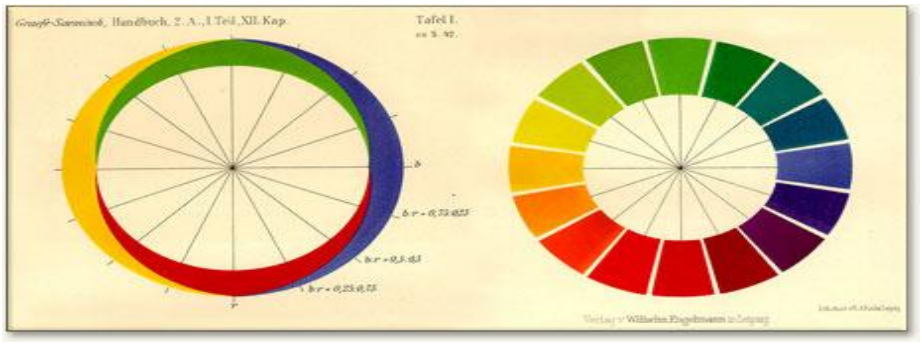

\section{PRACTICAL USES OF COLOURS}

Colour can be used in many different ways within a design. It cam highlight specific information that might oyherwise have been lost, it can draw attention, it can make the viewer feel compassion, love or hate, it can suggest femininity or masculinity and it can provide a cultural key to interpret and receive information. Colour presentys the designer with limitless opportunities. The use of colour within a design will usually require careful planning. If a publication dose not print with four colours, any colour fall or usage will normally be restricted to certain section sections in order to minimise costs. Use of an imposition plan will help a designer to determine the colour fall and placement and placement of special colours or varnishes within a printed publication.

There are several different methods of colour detailing that a designer can use to enhance a piece of work. These range from the use of surprints, overprints, tints and special colours, to the use of different paper stocks and print-finishing techniques such as foil stamping or fore-edge printing.

Colour Fall - Planning the incorporation of colour in a printed publication can be approached in a number of different ways. Most often colour usage is managed for an entire publication during its planning stage. This is achieved via an imposition and colour fall plan that shows which pages will be printed in different colours. 


\section{INTERNATIONAL JOURNAL of RESEARCH -GRANTHAALAYAH

Colour detailing - Colour can provide effective enhancement to a design because it grabs the attention of the viewer. Colour detail can also be included in the format or technical specifications of a publication.

Colour Layers - There are three techniques that can be employed to combine or layer printed fore and background elements, such as type and an image, these are surprint, reverse out and overprint. These techniques may be used to optimize visual appearance and clarity where elements overlap or to produce interesting graphic effects.

Surprint: A surprint is defined as two elements which print on top of each other and that are tints of the same colour.

Reverse Out : A reverse out removes parts of a flood colour, which apace in the shape of a given design, letters of characters.

Overprint: An overprint describes two elements that are printed one on top of the other, usually a darker colour is printed over a lighter colour.

Tints - The reproduction of colour is achieved by screening the three trichromatic process colours: cyan, magenta and yellow - usually in increments of 10\%. There are 1330 available tints from these three process colours, and many more are obtainable by incorporation black as well.

\section{USE IN MEDICINE AND THERAPY}

Colors are sometimes used in therapy (Also called Chromo therapy). Colors have a huge effect on people who have brain disorders or who are emotionally troubled. The color blue has a calming effect on many people and owers respiration and blood pressure. Red has the opposite effect. Some therapists use green to sooth and relax emotionally disturbed people who suffer from anxiety or depression. Some claim that the color violet is good for migraines and in cases of cellulites caused by a poor elimination, heaviness or sluggishness after eating, disorders of the spleen, bladder and kidney. Yellow helps energize people and relieves depression

\section{TECHNIQUES}

So far we have looked at how colours can be selected, classified and reproduced. Colour, however, is not only used for the cultural or symbolic values that it imparts, but is frequently used to embellish different components, homnogenise a disparate set of images or to add a hierarchy to the different textual elements used in a design. An understanding of some of the basic colouring techniques - such as toning, contrast, gradients, manipulation and hierarch - allows a designer to add an intelligent structure and cohesion to a design that it might otherwise be lacking. The images and text can be coloured to produce more striking, meaningful and consistent results. The ability to manipulate design elements through the use of colour allows the designer to better communicate their intended meaning.

Colouring images - A designer can colour an image through use of duotones, filters or a number of other techniques, colouring an image can impart element of drama to a design or suggest a certain style, perhaps radically changing it, either by accentuating or diminishing specific details. Tonal Images - A tonal image is akin to a black and white photograph in which the white tones have been replaced by another colour. Usually tha replacement will be one of the process colours, 


\section{INTERNATIONAL JOURNAL of RESEARCH -GRANTHAALAYAH

but any colour can be used. A duotone is a tonal image that id produced using two colours. The uses of tones creates visual uniformity between images as all coloured detail is reduced to tones of a single colour that is universally applied. This simplifies a print job because the number of colours that need to be printed are reduced.

Colour Manipulation - Using a limited palette of controls, it is possible to alter the appearance of an image, from subtle colour adjustments to more startling graphic intervention.

Levels : Levels refer to the distribution of tones within the image area. They can be adjusted precisely to select where the darkest and lightest points within an image will be. Levels allow us to control the black point the white point and the mid grey setting.

Curves : Curves map the brightness values of an image as such they allow a designer to make a pixel brighter or darker. Curve adjustments can be used to improve the contrast of an image or to make a creative intervention that borders upon abstraction.

Brightness and Contrast : The brightness and contrast control allows a designer to manage how light or dark an image is. Adjusting these values can add shade or hightlights to an image.

Hue and Saturation: These values allow a designer to change the colour of the whole image or a highlighted part of it. Hue selection alters the actual colour while saturation adjusts the depth of colour.

Colour Balance : Colour balance can be used to make adjustments across a wide range of colour creation.

Channels : A channels is an individual colour that makes up an image. Many images use the same combinations of channels but there is no need to be restricted to these.

Experimentation : Most techniques applied to the images on this spread are used as part of an overall image production process and mot in isolation. Experimenting with combinations of different techniques produces more creative results.

Gradients - This is a type of image fill that allows specific colours to be applies to a bitmap image which creates a transitional effect that flows from on colour to another. This effect can be used to provide a uniform treatment to various photographic elements by reducing them to coloured silhouettes or outlines. The application of a gradient can produce a number of creative possibilities. Undercolour Addition - The black produced by the black printing plate can look pale and washed out. To overcome this large areas of black often have a 50 to $60 \%$ cyan bouncer which will improve its appearance and colour depth.

Hierarchy - A hierarchy is a logical, organized and visual guide which is used for the text headings that normally accompany body text. Hierarchy, in this context indicates different types of content through variations in points sizes and styles. Colour can be used to establish a hierarchy. A piece of text when set as a solid block can be hard to navigate. Colour can be a useful tool to demarcate different levels of content importance or hierarchy. Typography typically uses titles and subheads to establish order. The examples shown here demonstrate that more experimental hierarchies can be employed.

A colour inderscore is used in conjunction with an indented paragraph to indicate the starting point. Highlighting the first word in piece of text is another device to denote the beginning.

\section{SYMBOL OF COLOUR}




\section{INTERNATIONAL JOURNAL of RESEARCH -GRANTHAALAYAH

Colour possess a wealth of symbolic meanings that are derived from cultural and social associations. Colour selections must therefore be made in consideration of the cultural norms and associations of the intended target audience, particularly if a design is to be seen internationally. As such those living in different countries and having different cultural values may vary in their reactions towards and connotations with the same colour. Colours hold significance for people around the world. Not only the colours influence emotion but they also hold meaning in religion and various cultures.

Red: Excitement, energy, passion, love, desire, speed, strength, power, heat, aggression, danger, fire, blood, war, violence, all things intense and passionate, sincerity. In Japan red colour symbolize happiness also.

Pink : Pink is a warm, exciting, fun and feminine colour. It symbolizes love and romance, caring, tenderness, acceptance and calmness.

Beige and ivory : symbolize unification. Ivory symbolizes quiet and pleasantness. Beige symbolizes calm and simplicity.

Yellow : Yellow is a bright and happy colour. It signifies joy, happiness, betrayal, optimism, idealism, imagination, hope, sunshine, summer, gold, philosophy, dishonesty, cowardice, jealousy, covetousness, deceit, illness, hazard and friendship.

Blue: Blue is a colour that alludes to the mysteries and power of the natural world, it is a colour of oceans and sky. It symbolize peace, tranquility, cold, calm, stability, harmony, unity, trust, truth, confidence, conservatism, security, cleanliness, order, loyalty, sky, water, technology, depression, appetite suppressant.

Dark Blue: Dark blue such as navy are considered conservative and uniform. It symbolizes integrity, knowledge, power, and seriousness.

Turquoise : Symbolizes calm. Teal symbolizes sophistication. Aquamarine symbolizes water. Lighter turquoise has a feminine appeal.

Purple: Purple is a regal colour which combines the hot tones of red with the cool tones of blue. It denotes royalty, nobility, spirituality, ceremony, mysterious, transformation, wisdom, enlightenment, cruelty, honor, arrogance, mourning, temperance.

Lavender : Symbolizes femininity, grace and elegance.

Orange: Orange is considered one of the hottest colour. It has been found to stimulate the emotion and appetite and denotes energy, balance, enthusiasm, warmth, vibrant, expansive, flamboyant, and demanding of attention.

Green: Green is a colour that embodies well-being mature and the environment- reflecting verdant fields and forests. It symbolize environment, healthy, good luck, renewal, youth, spring, generosity, fertility, jealousy, service, inexperience, envy, misfortune, vigor.

Brown: Broun is a neutral and down to earth colour. It represent earth, stability, hearth, home, outdoors, reliability, comfort, endurance, simplicity, and comfort.

Gray: Gray is a neutral colour. It denotes security, reliability, intelligence, staid, modesty, dignity, maturity, solid, conservative, practical, old age, sadness, boring. Silver symbolizes calm.

White: White is a neutral colour that provides a good contrast for a number of strong colours including black. Orange and blue. It is associated with reverence, purity, birth, simplicity, cleanliness, peace, humility, precision, innocence, youth, winter, snow, good, sterility, marriage (Western cultures), death (Eastern cultures), cold, clinical. 
Black: Black is quite simply the negation of colour. It symbolize Power, sexuality, sophistication, formality, elegance, wealth, mystery, fear, evil, unhappiness, depth, style, sadness, remorse, anger, anonymity, underground, good technical color, mourning, death (Western cultures), austerity, detachment.

\section{REPRESENTATIVE USES OF COLOUR}

Colour is used in many ways to effectively communicate different meanings. Colour can provide a powerful impact even when used in a very simple ways.

Emotions: Blue is seen as conservative. Red is power and aggression. Brighter colors such as yellow and orange represent warmth not only with emotions but also with temperature. Cool colors are blue, green, black or any color with a dark shade. When someone is feeling down or depressed, it is said they are feeling "blue." When someone is angry they "see red." When someone is seen to be afraid or "chicken" they are called yellow.

Ecology: Obviously green is the major color symbolizing ecology. The new phrase for people or companies who find ways to cut back on electricity, fuel, or things that damage the environment is "going green".

Phrases: When something is seen as opposite, extreme, or a firm position, it is said to be "black or white." When something is not clear or not in a firm position, it is said to be a "gray area." The blues describe a form of music.

Religion: Colours are also used in religious ceremonies or represent aspects of religion. Native Americans include colors in religious ceremonies. The Navajo Nation considers four colors to be important: Turquoise, white, yellow, and black. These colors represent four sacred mountains. The Apache Nation also considers four colors to be important: Green, white, yellow and black. These are sacred colors of the White Mountain and are also used in government. The Iowa Nation also considers four colors to be sacred: Black, yellow, red and white. They represent direction, their flag, and what they consider to be four races of man.

In Tibetan Buddhism, blue is the color of Vairochana, a celestial Buddha, whose image is the immensity of sky blue. Buddhist monks wear orange (specifically the color saffron) robes primarily due to tradition. That was the least expensive color dye at the time and that is what they continued to wear. The robes themselves symbolize "simplicity and detachment of materialism." Green is the traditional color of Islam. The Islamic flag is green. Green is also mentioned in the Quran as the color of garments, cushions and carpets in paradise

In Hinduism, saffron is their most sacred color. Saffron represents fire that burns our impurities. Yellow represents knowledge and learning. The color green of the Maharashtra represents life and happiness. The color blue is like infinity like the vastness of the oceans and sky

In Christianity, the color red symbolizes the blood of Jesus Christ and of sacrifice. White represents the body of Christ. Black represents sin in Catholic liturgy. Gray is the color of ash and this represents repentance in Catholicism. Purple is the liturgical color for the seasons of Advent and Lent. Heaven is described as having a lot of gold in buildings and streets. White and silver are used in liturgy during Christmas and Easter. The Bible says that many in the Middle East and Rome valued colored gems and jewelry. Red and white coral was used for beads and ornaments. Red rubies and light blue turquoise were given as gifts. (Ezekiel 27:16) 


\section{INTERNATIONAL JOURNAL of RESEARCH -GRANTHAALAYAH \\ A knowledge Repository}

Art

Colour Coding : A color code or colour code is a system for displaying information by using different colors. In this context colour coding is means to organize different types of information so that the various groups of content are instantly recognizable which helps us to quickly process the material we are viewing. Colour coding is widely used for example it can be found on clothing lables to indicate size, on traffic sings and even as a measure of national security status.

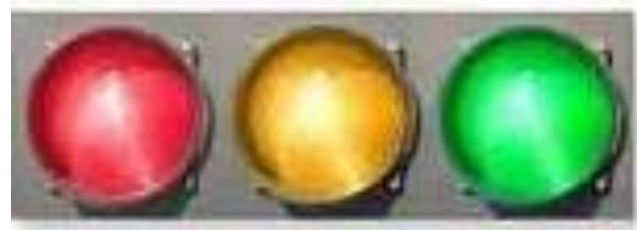

Colour as a warning : Colour can also provide an important form of alert: advertising danger. The bright colours of some species give predators a warning that they are likely to be unpalatable at best and poisonous at worst.

Red is a strong and stimulating colour. Red has high visibility and is therefore often used on warning signs or as an alert to potential danger. When red is used in this manner it allows the viewer to estimate the level of danger or risk and respond accordingly.
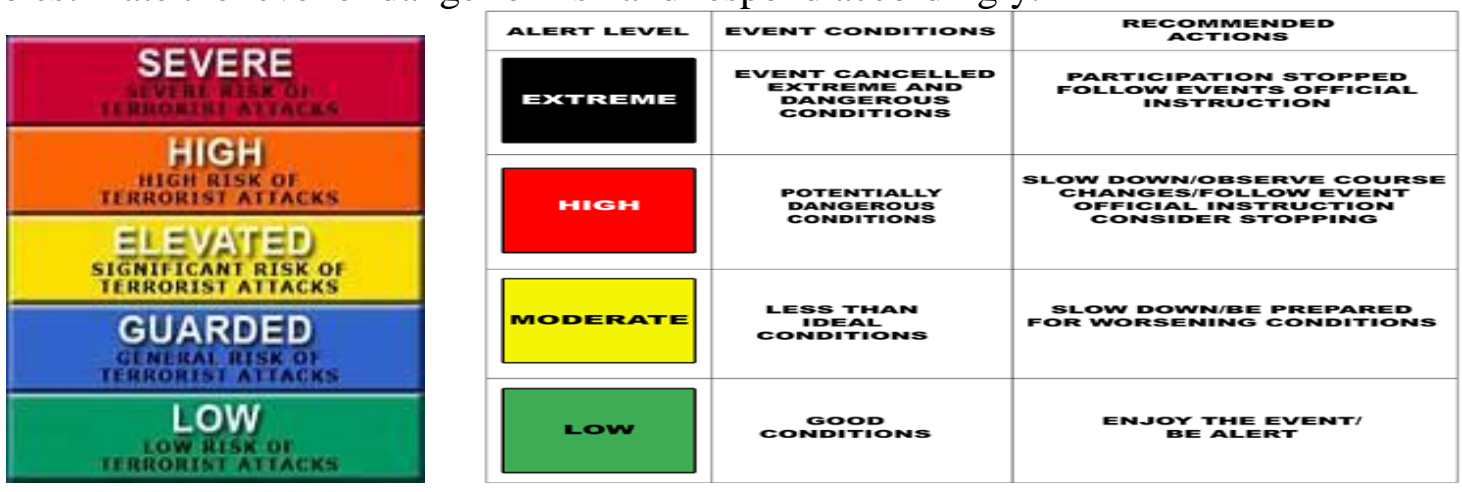

Creating Identity : Colour is often used to help establish a strong and instantly recognizable identity for a huge variety of organizations and their associated products or causes. Colour is used to condition and pre-empt the way in which people view the organization or their associated produce or work. Dark blues are used to create conservative, dependable and trustworthy identities for banks and insurance companies; bright primary colours are used to create identities for children's products, and pink is often used to create identities relating to beauty, love and sex.

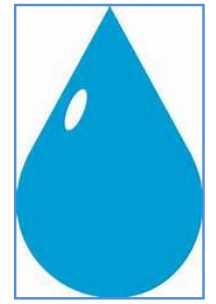

Water

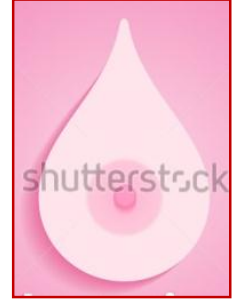

Milk

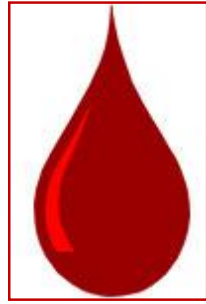

Blood

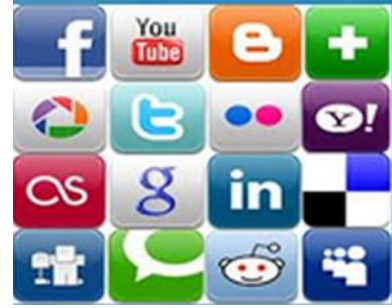




\section{INTERNATIONAL JOURNAL of RESEARCH -GRANTHAALAYAH \\ A knowledge Repository} Art

Colours in language - The language contains many idioms related with colours as - blue eyed boy, white lie, grey matter, red tape, blue/white collar worker, green fingers, give the green light, caught red handed, purple prose, blue chip, blackout, feeling blue, red - hot news, yellow belly, scarlet lady etc. Although no true meaning can be understood from the individual elements of the phrase the colour in the expression often hints at the intended meaning. The different associations we have made with each colour have been absorbed into our language, so they are frequently used to reinforce the emotion or mood someone is trying to convey.

\section{REFERENCES}

1 Hans Schwarz: Colour for the Artist, Wetson - Guptill Publication 1975

2 Ambrose \& Harris : Basic Design 05 COLOUR, AVA Book Publication. 2005

3 Frank M. Young : Visual Staudies . A Foundation for Artists and Designers, Whitehall Books limited 1985

4 Norman Buchanan : Painting and the Joy of Colour, Lowe \& Brydone Printers Ltd. 1968

5 Arthur L. Guptill : Colour in Sketching \& Rendering, Aravali Books International 2004

6 http://www.webexhibits.org/colorart/color.html

7 http://www.artiscreation.com/Color\%20of\%20Art.html

8 http://www.colormatters.com/color-and-design/basic-color-theory

9 http://www.incredibleart.org/lessons/middle/color $2 . \mathrm{htm}$ 